\title{
Response to Letter to the Editor: "Reproducibility of flow and velocity parameters in intracranial arteries measured with phase-contrast magnetic resonance imaging; a methodological issue"
}

\author{
${\text { Maria Correia de } \text { Verdier }^{1} \text { • Johan Wikström }}^{1}$
}

Received: 13 June 2016 / Accepted: 15 June 2016 /Published online: 22 July 2016

(C) Springer-Verlag Berlin Heidelberg 2016

\section{Dear Sir,}

Thank you for the opportunity to respond to the comments on our article [1]. As Dr Sabour pointed out in the Letter to the Editor, the correct method when evaluating reproducibility using intra-class correlation coefficient (ICC) in our study is to calculate ICC for single measurements and not for mean of measurements. We used ICC1 (single rater, absolute agreement; calculated as ICC $(1,1)$ described by Shrout and Fleiss [2]).

As we used a method for testing repeatability for single measurements, we believe that our conclusion is valid, i.e., reproducibility for $2 \mathrm{D}$ phase-contrast magnetic resonance imaging (PC-MRI) measured flow and velocity parameters is overall quite low, but higher for middle cerebral artery than for posterior cerebral artery and anterior cerebral artery.

Compliance with ethical standards

Conflict of interest We declare that we have no conflict of interest.

\section{References}

1. Correia de Verdier M, Wikstrom J (2016) Normal ranges and testretest reproducibility of flow and velocity parameters in intracranial arteries measured with phase-contrast magnetic resonance imaging. Neuroradiology 58(5):521-531. doi:10.1007/s00234-016-1661-6

2. Shrout PE, Fleiss JL (1979) Intraclass correlations: uses in assessing rater reliability. Psychol Bull 86(2):420-428
This comment refers to the article available at 10.1007/s00234-016$1717-7$

Maria Correia de Verdier

maria.correiadeverdier@radiol.uu.se

1 Department of Radiology, Uppsala University, Uppsala University Hospital, SE-751 85 Uppsala, Sweden 